\title{
Acid hydrolysis of spent coffee grounds: effects on possible prebiotic activity of oligosaccharides
}

Fabrizio Sarghini ${ }^{1}$, Francesco Marra ${ }^{2 *}$, Angela De Vivo ${ }^{1 *} \mathbb{C}$, Paola Vitaglione ${ }^{1}$, Gianluigi Mauriello ${ }^{1}$, Diamante Maresca' ${ }^{1}$ Antonio Dario Troise ${ }^{1,3}$ and Esteban Echeverria-Jaramillo ${ }^{4}$

\begin{abstract}
Background: Spent coffee grounds (SCG) are a promising source of natural by-products which can be used for different purposes. In this work, a possible use of oligosaccharides isolated from SCG as functional ingredients was investigated. SCGs were treated with an acid hydrolysis at high temperature $\left(200^{\circ} \mathrm{C}\right)$ in a closed reactor setting reaction time of 30,60 and $90 \mathrm{~s}$ depending on the sample (original or defatted). A comprehensive study of the resulted watersoluble hydrolysate using a high-resolution mass spectrometry analysis was performed. Additionally, the growth of four Lactobacillus strains was tested to assess the prebiotic potential of the hydrolysate.
\end{abstract}

Results: Oligosaccharide chains formed by hexoses with a degree of polymerization ranging from 3 to 6 were identified and characterized. Regardless of the composition and the reaction time of hydrolysis, the bacterial activity of SCG extracts exhibited significantly higher values than the well-known versatile carbohydrate used by food industry, i.e., inulin.

Conclusions: The results pave the way toward the use of hydrolysate SCG as an innovative ingredient intended to fortify food formulations. The diversity in coffee oligosaccharides composition suggests their selective prebiotic activity for specific bacterial strains.

Keywords: Spent coffee grounds, Functional oligosaccharides, High-resolution mass spectrometry (HRMS), Prebiotic, Industry by-product

\footnotetext{
*Correspondence: fmarra@unisa.it; angela.devivo@unina.it

${ }^{1}$ Department of Agricultural Sciences, University of Naples Federico II, 80055 Portici, NA, Italy

2 Department of Industrial Engineering of the University of Salerno, 84084 Fisciano, SA, Italy

Full list of author information is available at the end of the article
} 


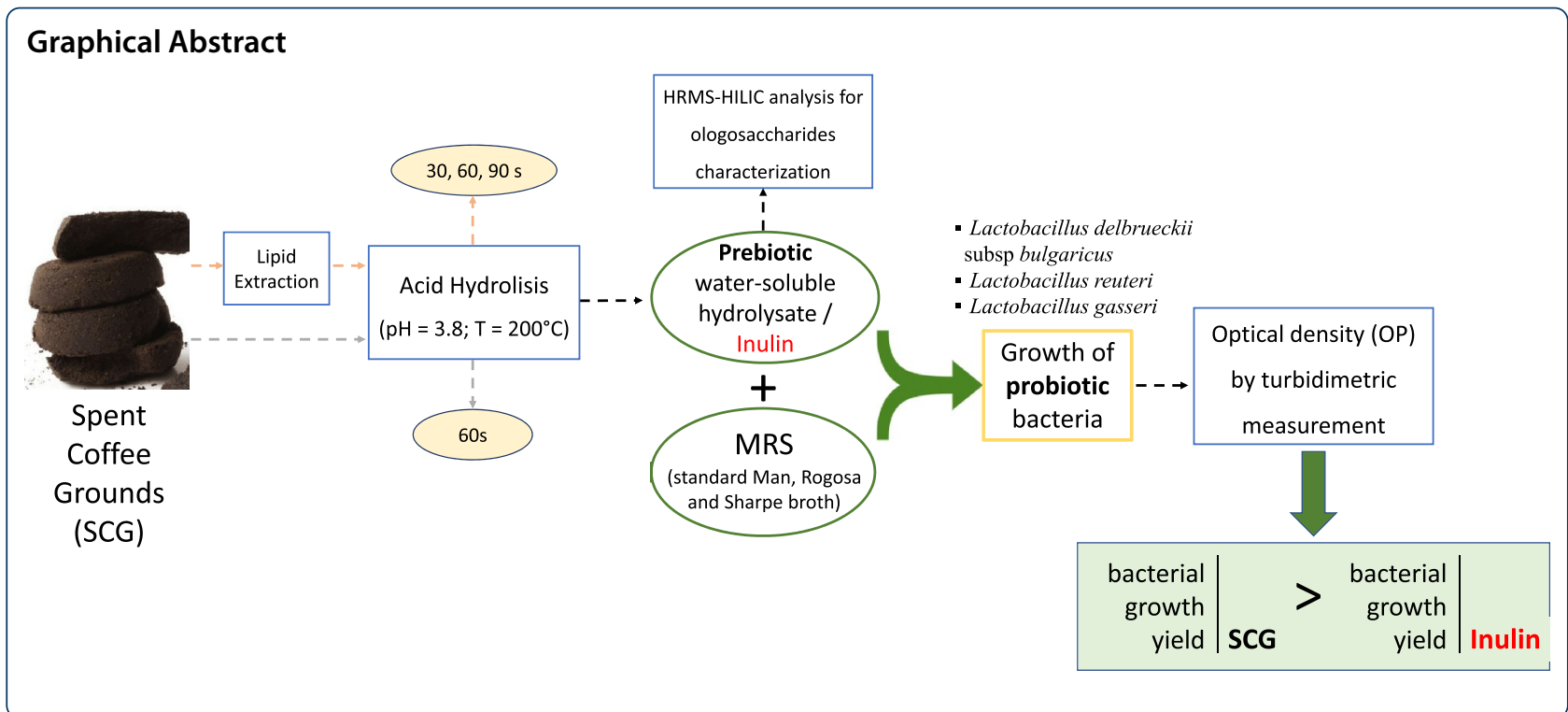

\section{Introduction}

Agricultural and food processing industries produce millions of tons of losses and waste products that can be released at different stages of food production, i.e., cleaning, processing, cooking, and/or packaging [1-4]. The characterization and the effective utilization of these by-products is important to reduce the environmental pollution of the food industry contributing to ensure the sustainable management of waste. In addition, the correct waste disposal implies a cost burden which is becoming a grave economic issue. The growing worldwide demand for healthy food components has pushed technological innovation to develop functional ingredients and/or new foods or natural additives in the context of the exploitation of the food by-products which, as is known, are rich in phenolic compounds, dietary fibers, polysaccharides, vitamins, carotenoids.

From this perspective, a few million cubic tons of highvalue solid residues are generated every year during coffee processing stages from harvesting to consumption [5]. The coffee husks/pulp are the main by-products of coffee agro-industry obtained when freshly harvest coffee cherries (berries) are processed to produce green coffee. Specifically, depending on the dry or wet method, solid residues are generated, respectively, as pulp or husk [6]. More than $50 \%$ of the coffee fruit is not used for production of the commercialized green coffee and, therefore, is discarded during processing [7].

Coffee silverskin is the only by-product of the roasting process and it is a thin tegument of the outer layer of the coffee bean. Spent coffee grounds (SCG) is referred to the residue produced during the extraction process by the combination of raw coffee powder with hot water or steam. SCGs are generated through industrial-scale brewing of coffee beans to produce instant or soluble coffee grounds with a worldwide annual generation of 6 million tons [8]. SCG contains approximately $13.5 \%$ protein, $2.3 \%$ fat, $72 \%$ of carbohydrate with $51 \%$ as insoluble dietary fiber and $10 \%$ as soluble dietary fiber $(\mathrm{g} / 100 \mathrm{~g}$ dry weight) and it also contains amounts of tannins, polyphenols, and caffeine $[5,9]$. The presence of these bioactive compounds, well known to exhibit toxic effects on the soil ecosystem prevents SCG from being disposed at landfills. Similarly, SCG cannot be incorporated into animal fodder because of the anti-nutritional activity of tannins $[10,11]$. Therefore, there is the need for safe disposal of SCG. From the composition of SCG it is clear that it could be used as a source of antioxidants and dietary fiber. These components could be used as a whole ingredient or could be added for nutritional enrichment of food products. Several reports have confirmed the utilization of SCG as a high value-added by-product/functional ingredient of foods. Mussatto et al. [8] established the optimal SCG hydrolysis working conditions to maximize the extraction of hemicellulose sugars which could be used as a substrate in fermentative processes.

Antioxidant dietary fibers isolated from SCG can be incorporate to bakery product such as biscuits with a positive impact on circadian rhythm of young adults as investigated by Oseguera-Castro et al. [12] or with antidiabetic potential as shown in the study of VázquezSánchez et al. [13] in which content of advanced glycation end products has decreased in fortified biscuits compared to the traditional ones. SCG also reportedly used directly as food ingredient to obtain high-quality innovative biscuits with the potential to reduce the risk of 
chronic diseases such as diabetes, aging and obesity [14]. Through a simulated gastrointestinal digestion process, Castaldo et al. [15] reported that the addition of SCG in the cookie dough improved the in vitro bioaccessibility of polyphenols, with an increase of about 10 percentage points of bioaccessibility in the colon phase compared to the control samples. Sampaio and Machado et al. [16, 17] demonstrated the successful use of SCG as raw material for the production of fermented and distilled novel beverages with smell and taste of coffee through a process comprising three main steps, i.e., a hydrothermal extraction of aroma compounds from SCG, fermentation and distillation.

Furthermore, high dietary fiber-rich SCG can be used a functional ingredient in food formulations to add prebiotic effect and thus for aiding the growth of the gut microbiota blocking attachment of pathogens. The ability of bacteria to utilize SCG as carbon source was demonstrated by Jimenez-Zamora et al. [11]. However, it has been reported that SCG with the presence of coffee melanoidins exerted a negative effect over beneficial bacteria, decreasing the numbers of lactobacillus and bifidobacterial and thus the isolation of melanoidins could help to control the concentration added to foodstuffs. It also highlighted the antimicrobial and antioxidant properties of SCG.

The characterization of structure of intact oligosaccharides and their monomeric composition in SCGs was performed in order to evaluate their prebiotic effect by using multiple chromatographic and spectroscopic analytical tools [18]. The proven heterogenous composition of each monosaccharide unit suggests that only specific probiotic bacteria possess the enzymes needed to break the glycosidic linkages of monosaccharides before they are used as a carbon source. Especially mannooligosaccharides derived from mannan in SCG has showed beneficial health effects on large bowel function including the promotion of growth of bifidobacteria as well as production of short-chain fatty acids by in vitro studies, in animal models, and in human studies [19-22]. Furthermore, the potential prebiotic role of mannooligosaccharides derived from hydrolyzed SCG, and the symbiotic activity with lactic acid bacteria has recently been investigated [23].

In view of the aforementioned, this research aimed at investigating the use of high-temperature acid hydrolysis in a closed reactor as a viable technology to obtain functional ingredients from SCG for application in human health as food supplements. More specifically, this study intends to characterize the type of carbohydrates present in the resulting hydrolysate and based on previous findings assess their viability as prebiotics on selected probiotic strains. As a control, the inulin was used since it has been recognized as dietary fiber displaying prebiotic activities making it a valuable ingredient in the formulation of functional food products [24].

\section{Materials and methods Chemicals}

Acetonitrile, methanol and water for liquid chromatography-high-resolution mass spectrometry (LC-HRMS) analysis were obtained from Merck (Darmstadt, Germany). Hydrochloric acid (37\%), n-hexane (>99\% purity $\mathrm{GC}$ ), sodium hydroxide, ammonium hydroxide (33\%), ammonium acetate, silicon dioxide, sorbitan monooleate, dipotassium hydrogen phosphate, sodium acetate, triammonium citrate, magnesium sulfate, manganese sulphate, the analytical standards stachyose, raffinose, maltose, glucose, and inulin (code I3754) were purchased from Sigma-Aldrich (St. Louis, MO). MRS (standard Man, Rogosa and Sharpe) broth was reconstituted according to the standard composition by replacing glucose with SCG hydrolysate or inulin. All the components of MRS broth were purchased from Thermo Scientific Oxoid (Peptone, Lab-Lemco powder and Yeast Extract).

\section{Sample preparation}

The spent coffee grounds (SCG) were obtained from mixtures of Arabica and Robusta coffee (ratio 70:30) from different origins provided by Kimbo S.p.A (Naples, Italy). The brewing of the beverage was carried out with 7 to $15 \mathrm{~g}$ ground roasted coffee in a commercial espresso machine (Rancilio Classe 10 USB 2, Italy) that operated at 1 bar and required approximately $24 \mathrm{~s}$ at $90{ }^{\circ} \mathrm{C}$ for each extraction. The initial moisture of the sample was measured as $53.97 \% \mathrm{w} / \mathrm{w}$ with a moisture analyzer (Radwag MA 50 Poland). The material was dried at $60{ }^{\circ} \mathrm{C}$ for $8 \mathrm{~h}$ in a stove with air circulation (MMM Group IP20, Germany) to final moisture of $3.69 \% \mathrm{w} / \mathrm{w}$. Subsequently, the samples were stored at $4{ }^{\circ} \mathrm{C}$ until further use.

\section{Lipid extraction}

Lipids were extracted according to Yen et al. [25] with some modifications. $50 \mathrm{~g}$ of the SCG were placed into a 1-L flask with $\mathrm{n}$-hexane with a 1:4 ratio (\% w/v). The sealed flask was agitated by a magnetic stirrer for $16 \mathrm{~h}$ at room temperature. Next, the mixture was separated by decantation and filtration using Whatman paper. This process was repeated two times. The recovered material was introduced in the oven at $60{ }^{\circ} \mathrm{C}$ for $3 \mathrm{~h}$ until the residual hexane was completely evaporated. Then the sample material was stored in a plastic containers at $-18^{\circ} \mathrm{C}$ until the extraction. 


\section{Acid-catalyzed extraction}

Acid-catalyzed extraction was adapted according to the methods of Fulger and Asano et al. [19-26]. The defatted spent coffee grounds were suspended in distilled water to form slurry at a concentration of $5 \%(\mathrm{w} / \mathrm{w})$ and its $\mathrm{pH}$ was adjusted to 3.8 with a $2 \mathrm{M}$ solution of hydrochloric acid. The slurry was then introduced in a reactor keeping the agitation constant. The reactor consisted of a stainless-steel cylindrical chamber with the following characteristics: external radius of $2.5 \times 10^{-2} \mathrm{~m}$, internal radius of $1.6 \times 10^{-2} \mathrm{~m}$, height equal to $1 \times 10^{-1} \mathrm{~m}$ and depth of $1.8 \times 10^{-2} \mathrm{~m}$. The reactor was preheated prior the addition of the slurry to $60{ }^{\circ} \mathrm{C}$ and subsequently sealed when full. Subsequently, it was introduced into heated purified silicon dioxide (sand) at $200{ }^{\circ} \mathrm{C}$. The reaction time was taken into account after the chamber and the slurry reached thermal equilibrium and consisted of 30, 60 and $90 \mathrm{~s}$ depending on the sample. Once the necessary time was reached, the chamber was removed from the sand and immediately cooled down on ice water to stop the reaction and prevent further hydrolysis of the polysaccharide chains. Next, the reactor was opened, and the slurry was neutralized with a solution $1 \mathrm{M}$ of sodium hydroxide until reaching $\mathrm{pH}$ 5.8. Subsequently, this solution was separated with a centrifuge (ALC 4232, Italy) at a speed of $3800 \mathrm{rpm}$ for $15 \mathrm{~min}$. The liquid part, or hydrolysate, was recuperated and stored for further characterization. In addition to the defatted SCG, a variation used SCG with its full fat content with a time of extraction of $60 \mathrm{~s}$ (60sfat) in order to evaluate the influence of lipids on the characteristics of the hydrolysate.

\section{High-resolution mass spectrometry (HRMS) detection}

The identification of oligosaccharides was performed according to the method of Bajad et al. [27] with some modifications. Supernatant collected from each sample $(10 \mu \mathrm{L})$ were analyzed by HRMS, while chromatographic separation was achieved by using a U-HPLC Accela system 1250 (Thermo Fisher Scientific, Bremen, Germany) consisting of a degasser, a quaternary pump, a thermostated autosampler $\left(10{ }^{\circ} \mathrm{C}\right)$ and a column oven set at $30{ }^{\circ} \mathrm{C}$. A Luna amino column $(100 \times 2.0 \mathrm{~mm}, 3.0 \mu \mathrm{m}$; Phenomenex, Torrance, CA) operating in HILIC mode and equipped with a security guard of the same stationary phase was used. Mobile phase A was acetonitrile while mobile phase $B$ was ammonium acetate $(3 \mathrm{mM}$, pH 9.2 adjusted with $30 \mathrm{mM}$ ammonium hydroxide). The following gradient of solvent B ( $\mathrm{min} / \% \mathrm{~B}):(0 / 15),(2 / 15)$, (6/100), (11/100) was used at a flow rate of $300 \mu \mathrm{L} / \mathrm{min}$. The autosampler needle was rinsed with $400 \mu \mathrm{L}$ of acetonitrile/water $50 / 50(\% \mathrm{v} / \mathrm{v})$ before each injection. To set up the optimal condition, an aqueous solution of stachyose and raffinose $(10 \mu \mathrm{g} / \mathrm{mL})$ was infused directly into the
Exactive Orbitrap HRMS system (Thermo Fisher Scientific, Bremen, Germany) equipped with a heated electrospray interface operating in both positive and negative mode and scanning the ions in the $\mathrm{m} / \mathrm{z}$ range of 60-200. The resolving power was set to 75,000 full width at halfmaximum (FWHM, $m / z 200$ ), resulting in a scan time of $1 \mathrm{~s}$. The automatic gain control was used to fill the C-trap and the maximum injection time was $50 \mathrm{~ms}$. The interface parameters were as follows: spray voltage, $3.2 \mathrm{kV}$; capillary temperature $290{ }^{\circ} \mathrm{C}$ and heater temperature at $350^{\circ} \mathrm{C}$, capillary voltage, $50 \mathrm{~V}(-55 \mathrm{~V}$, for negative ions); skimmer voltage, $14 \mathrm{~V}(-34 \mathrm{~V}$ for negative ions); tube lens voltage $190 \mathrm{~V}$ ( $-190 \mathrm{~V}$ for negative ions); sheath gas flow, 45 (arbitrary units); and auxiliary gas flow, 9 (arbitrary units). Before the oligosaccharides quantification, the instrument was externally calibrated by infusion with a positive ions solution that consisted of caffeine, MetArg-Phe-Ala (MRFA), Ultramark 1621, and acetic acid in a mixture of acetonitrile/methanol/water $(2: 1: 1, \% \mathrm{v} / \mathrm{v} / \mathrm{v})$, then with a negative ions solution that consisted of sodium dodecyl sulfate, sodium taurocholate, Ultramark 1621 , and acetic acid in a mixture methanol/water (1:1, $\% \mathrm{v} / \mathrm{v})$. To optimize the HRMS conditions and the mass accuracy, the instrument was calibrated each day both in positive and negative mode. A stock solution of stachyose and raffinose was prepared by dissolving $10 \mathrm{mg}$ of standard in $1 \mathrm{ml}$ of mass spectrometry grade water. The solution was diluted and stored at $-20^{\circ} \mathrm{C}$ until use. The range of the calibration curve was between 10 and $1000 \mathrm{ng} / \mathrm{ml}$ according to the limit of detection (LOD) and the limit of quantitation (LOQ). Three replicates of $1 \mathrm{ng} / \mathrm{ml}$ solutions were injected into the U-HPLC-HRMS system to verify the lowest concentration for which the signal-tonoise ratio was $>3$. Concentrations of $1 \mathrm{ng} / \mathrm{ml}$ resulted in no signal. The LOQ was $5 \mathrm{ng} / \mathrm{ml}$ for the standard solution, while the LOD was five times lower, and the $\mathrm{r}^{2}$ value was $>0.99$ in the above-mentioned range. Reproducibility of the method was evaluated through the intraday and inter-day assay. The slope among the three subsequent calibration curves showed a \% RSD of $<10 \%$. Control samples consisted of SCG before the hydrolysis process. They were spiked with a solution of stachyose (5000 ng/ $\mathrm{ml}$ ) and the recovery (R) was calculated according to the following formula:

$$
R=\left(\frac{C_{a}}{C_{s}}\right) \times 100,
$$

where $C_{a}$ is the concentration of the spiked analyte in the samples and $C_{s}$ is the concentration of the standard in water. 


\section{Functionality assessment}

Microbiological studies on common prebiotic strains were carried out in order to assess the functionality of each of the extracts that were obtained. These mainly consisted in the evaluation of microbial growth of the selected bacteria in different mediums in which the source of carbohydrates was replaced by the SCG hydrolysate. More specifically, the parameters for this experiment included the use of four strains of lactobacilli with prebiotic potential, the preparation of six different growing mediums, the conditions for fermentation applied uniformly to all of them and the method of quantification of the bacterial growth after fermentation. The details are explained in the following section.

\section{Bacterial strains and culture conditions}

Four Lactobacillus strains were used, a commercial strain of Lactobacillus delbrueckii subsp bulgaricus isolated from the food supplement Yovis in a previous research [28] and here named YOV5; the commercial strain Limosilactobacillus reuteri DSM 17938 gently provided by BioGaia (BioGaia AB, Stockholm, Sweden) and here named REU; the strains AL3 and AL5 belonging to the species Lactobacillus gasseri, previously isolated by Maresca et al. [29]. YOV5 and REU were identified by sequencing of $16 \mathrm{~S}$ rRNA gene, while AL3 and AL5 were identified by whole genome sequencing [30]. Strains were routinely grown overnight at $37{ }^{\circ} \mathrm{C}$ in MRS broth (Thermo Scientific). The concentration of each culture was standardized in order to yield an $\mathrm{OD}_{650}$ of 1.5. Subsequently each one was diluted in a buffer solution at $\mathrm{pH}$ 7.0. After this procedure, they were ready for use in the different media.

Medium preparation Six different MRS broths were prepared. Each varied from the other in the source of carbohydrates. MRS 5 served as control and corresponded to a standard formulation of the broth with glucose. In contrast, MRS 1 to 4 replaced glucose with a fixed volume of the SCG hydrolysate from the four different extraction conditions. Finally, MRS 6 replaced glucose with inulin in order to assess the behavior of the chosen bacterial strains when their source of carbohydrates for development is a commercial food grade prebiotic oligosaccharide.

Fermentation A fresh liquid culture of each strain was washed twice in phosphate buffer solution $\mathrm{pH} 7.0$ and resuspended to reach an $\mathrm{OD}_{650}$ of 1.5. A volume of $100 \mu \mathrm{L}$ was used to inoculate in triplicate test tubes that containing $10 \mathrm{~mL}$ of the six different broths. These tubes were incubated (universal oven Memmert UN160, Germany) at $37^{\circ} \mathrm{C}$ for a period of $24 \mathrm{~h}$.
Microbial growth The bacterial growth was evaluated by a turbidimetric method. It consisted in measuring the optical density (OP) of each sample with the use of a spectrophotometer (Eppendorf Bio Spectrometer Basic, Canada) at $650 \mathrm{~nm}$. For each different variation, a medium without bacterial growth was used as the standard to calibrate the blank.

\section{Statistical analysis}

Within the characterization and the functionality tests, the injections and the turbidity essays, were performed in duplicate and triplicate, respectively. The means and standard deviations were calculated. Subsequently, they were subject to a one-way analysis of variance (ANOVA) and the Tukey method was used as multiple mean comparison post hoc test. All the differences among the means were declared using $p \leq 0.05$ as the interval of confidence. The calculations were performed with Microsoft Excel (Microsoft Corporation, 2010, USA) and XLstat (Addinsoft, 2014, USA).

\section{Results and discussion}

\section{Composition of the extracts}

The hydrolysis procedure aimed to partially cleave the long polysaccharide chains present in SCG to reduce their degree of polymerization (DP) from the average of 45 and molecular weight of $7.3 \mathrm{kDa}$ to chains with 3 to 10 sugar residues [31]. The ultimate goal consisted in obtaining galactomannooligosaccharides from the predominant galactomannan fraction of the hemicellulose component left in coffee after brewing, without extending the degradation of the chains to the extent of obtaining only monosaccharides. For this reason, all conditions were kept constant except for the time of reaction, which was set at 30,60 and $90 \mathrm{~s}$. The DP will be further discussed in the next section.

Although a small fraction of galactomannans and arabinogalactans are extracted at coffee roasting and during the beverage preparation, most of these polysaccharides remain as insoluble material bound to the SCG matrix [8]. Even though this SCG is rich in polysaccharides, mainly galactomannan, crosslinked with proteins and lignins, their isolation without the application of an additional treatment is difficult [32]. Therefore, it was necessary to aggressively cleave any of these links with the use of very high temperatures $\left(200{ }^{\circ} \mathrm{C}\right)$ and acid $(\mathrm{pH} 3.8)$ in combination.

\section{Mass spectrometry analyses}

Table 1 shows HILIC-HRMS characterization of the extracts obtained after hydrolysis on the SCG. The $\mathrm{m} / z$ ratio and retention time of the extracts showed a degree 
Table 1 Identification of the oligosaccharide products identified in the extracts by HRMS performance based on the theoretical and experimental values of $\mathrm{m} / \mathrm{z}$. The optimization and quantification were optimized by using stachyose and raffinose

\begin{tabular}{llccc}
\hline Number of hexoses & Oligosaccharide products & $\mathbf{m} / \mathbf{z}$ theoretical & $\mathbf{m} / \mathbf{z}$ experimental & $\begin{array}{c}\text { Retention } \\
\text { time (min) }\end{array}$ \\
\hline 3 & Trisaccharide & 503.16 & 503.16 & 5.5 \\
4 & Tetrasaccharide & 665.21 & 665.22 & 5.3 \\
5 & Pentasaccharide & 827.23 & 827.27 & 5.1 \\
6 & Hexasaccharide & 989.32 & 989.32 & 5.3 \\
7 & Heptasaccharide & 1151.37 & Not found & - \\
\hline
\end{tabular}

of in the evaluation of microbial growth ofpolymerization that varied from 3 to 6 units.

As seen in Fig. 1, for the four extracts the tendency was to yield a high amount of trisaccharides $(>70 \%)$ followed by tetrasaccharides $(15-20 \%)$ and low quantities of pentasaccharides $(4-7 \%)$ and hexasaccharides $(<1 \%)$. The total carbohydrate composition of SCG has been reported to be between 60.3 and $65.9-\mathrm{g} / 100 \mathrm{~g}$ SCG dry weight [33-35] showing that galactomannan is the main polysaccharide ( 40 to $50 \%$ ) followed by arabinogalactan (18\%) and small part of cellulose and other minor sugars (2\%) [36]. Galactomannans consist of a main chain of $1 \rightarrow 4$ linked mannan with galactose unit side chains linked at C-6 with different degrees of branching. It is has been also established that in a polymeric structure the side chains are released first followed by the cleavage of the main chains $[8,31,37]$. Based on this evidence, it is highly probable that we have obtained oligomers from the cleavage of the galactomannan polymer at sites where the chain was branched with galactose as well as at sites where there was not a substitution. This further exemplifies the fact that it is common to find isomeric structures when analyzing galactomannan hydrolysis.

Previous research identified galactomannans oligosaccharides by flow injection mass spectrometry experiments as the sodium adducts $[\mathrm{M}+\mathrm{Na}]^{+}$of $2 \mathrm{Hex}$ and 3 Hex followed by 4 Hex at $m / z 365, m / z 527$ and $m / z 689$, respectively [38-40]. Although the detection method differs from the one used on this study, which is based on a negative ion rather than the investigation of sodium adducts formation, the optimization of alkaline HILIC conditions and HRMS experiments by using stachyose and raffinose allowed to characterize hexoses fractions (Table 1). Similar findings were also obtained for the characterization of melanoidins in coffee beans by using MALDI-ToF approach [41].

Table 2 shows a comparison of the products obtained in this experiment to ones reported by other authors under similar conditions $[19,20,26,42]$. The tendency of obtaining a mixture of oligosaccharides with various degrees of polymerization is the same. However, it is worth remarking that only under the conditions in this study, batch reactor with hydrochloric acid, more trisaccharides and less hexasaccharides were produced. This leads to infer that this procedure achieves a further hydrolysis of the galactomannan chains. This can be put in context with the conclusions reached by Fulger et al. [26] that state that harsher conditions favor the production of oligomers with lower degrees of polymerization, reaching the limiting case of producing mannose, while milder conditions favor a solution with a distribution of oligomers having higher degrees of polymerization. Although this tendency is seen in all the mentioned experiments, this principle does not apply completely when comparing the current procedure against the ones performed by the other authors [19, 20, 26, 42]. Within similar conditions, they do not achieve to obtain high polymerized products, thus a batch reaction with hydrochloric acid, promoted a harsher environment than a continuous reactor with other kind of acids; in acidic conditions the protonation of amino groups in SCG hindered glycation reactions favoring the release of oligosaccharides. In terms of the amount of oligosaccharides identified and their composition, the sample without removal of lipids and subject to a 60 -s extraction (60sfat), yielded significantly lower amount of carbohydrates and had a different composition compared to lipid free samples. With regard to its composition, 60sfat is significantly different from the other samples a lower amount of hexoses with a degree of polymerization of three, and a higher amount of $4 \mathrm{Hex}-\mathrm{a}$ and hexasaccharides. The other three extracts namely, $30 \mathrm{~s}, 60 \mathrm{~s}$ and $90 \mathrm{~s}$, did not present considerable differences among them with regard to the amount of oligosaccharides extracted as well as on their composition (Fig. 1) that is mainly trisaccharides surrounding $78 \%$, tetrasaccharides around $16 \%$ and almost negligible hexasaccharides $(<0.4)$. The profile of sample 60sfat shows significantly lower quantities of simple sugars favoring the presence of longer chain molecules, as well as a relatively lower yield compared to 60sfat. Consequently, it can be concluded that the presence of fat in the SCG caused an interference on the extraction, 


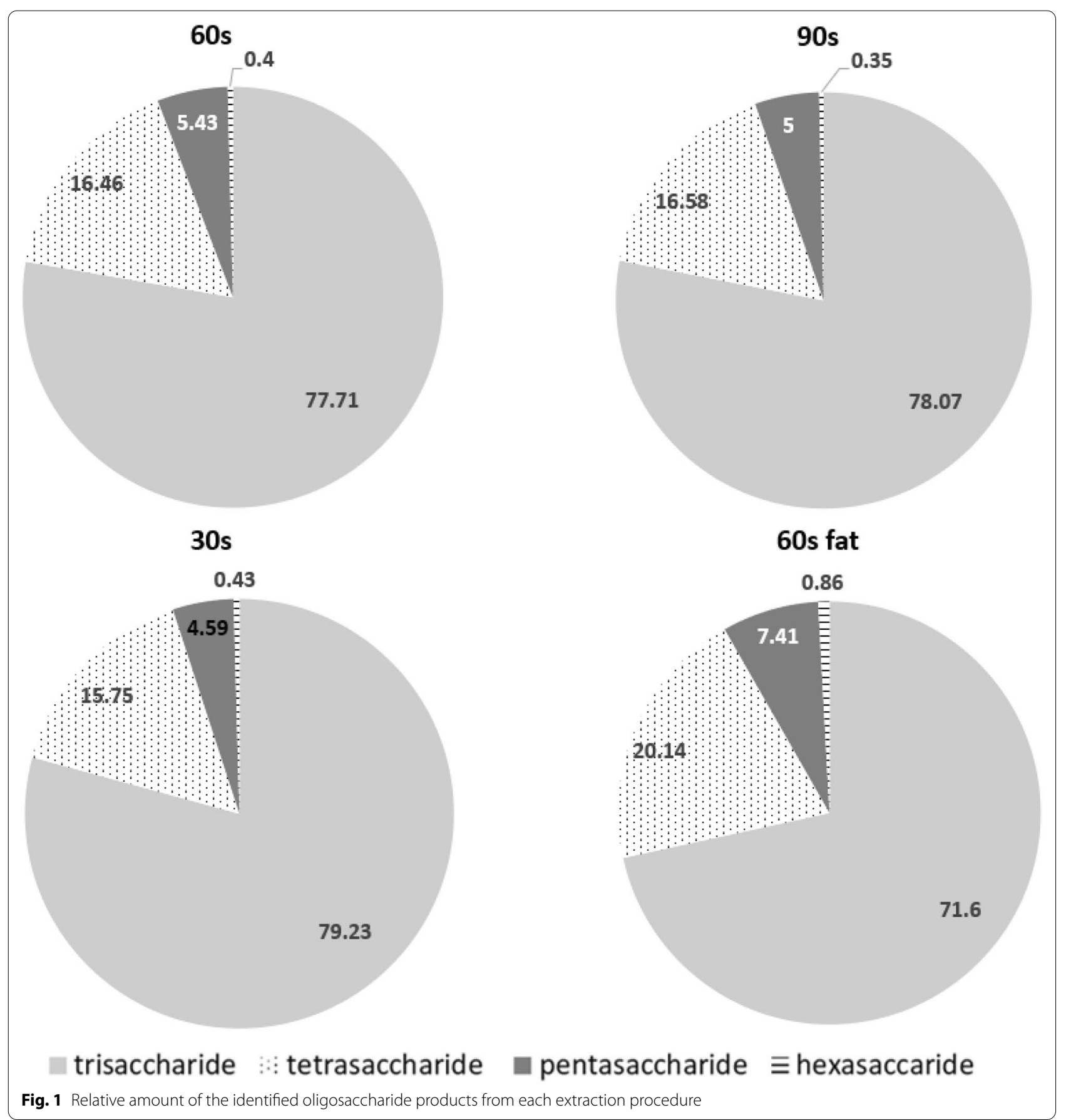

yielding longer chains which also limited the concentration of oligosaccharides obtained and reduced the degree of cleavage of the polysaccharide chains. The lipid fraction, which accounted for a $15-20 \%$ of the total dry weight of the SCG, influenced the reactivity of the sample by protecting the SCG from the hydrolysis. It could be hypothesized that the presence of lipids and complex oligosaccharides chains generated supramolecular structures that reduced the depolymerization; thus, the energy required must be higher to achieve the same degree of hydrolysis when comparing to SCG whose fat has been removed.

\section{Functionality}

The extracts were subject to a functionality test based on their capacity to enhance the growth of probiotic 
Table 2 Comparison of the oligosaccharide products obtained by this experiment and other authors among different extraction procedures with similar conditions at $200{ }^{\circ} \mathrm{C}$ and $30 \mathrm{~s}$

\begin{tabular}{lllll}
\hline Procedure & Trisaccharides (\%) & Tetrasaccharides (\%) & Pentasaccharides (\%) & $\begin{array}{l}\text { Hexasaccharides } \\
\text { (\%) }\end{array}$ \\
\hline Fulger et al. [26] & 43.31 & 33.12 & 13.38 & 10.19 \\
Fulger et al. [26] & 37.26 & 27.84 & 20.99 & 13.92 \\
Fulger et al. [26] & 21.37 & 23.28 & 25.95 & 29.39 \\
Takao et al. [42] $220{ }^{\circ} \mathrm{C}$ & 34.18 & 26.58 & 21.52 & 17.72 \\
Asano [19, 20] $220{ }^{\circ} \mathrm{C}$ & 44.26 & 31.15 & 16.39 & 8.20 \\
Current study & 79.23 & 15.75 & 4.59 & 0.43 \\
\hline
\end{tabular}

bacteria. Humans lack the enzymes to digest short chains of sugars as opposed to intestinal bacteria that can take advantage of them for their metabolic functions [43]. Additionally, Asano et al. [19] have performed in vitro digestion studies on tri to hexasaccharides of mannose proving that they constitute indigestible oligosaccharides since they were not decomposed by human salivary $\alpha$-amylase after $4 \mathrm{~h}$ of incubation showing resistance to gastric juice and to intestinal enzymes. These oligosaccharides were able to reach the large intestine without digestion thus constituting a potential substrate source for probiotic bacteria. Lactobacilli strains (AL3, AL5, Reu and Yov5), potential probiotic bacteria [44-49], were evaluated in their usual laboratory growth medium with the substitution of the carbon source from each of the extracts as well as with inulin to contrast their behavior with a common and well-studied prebiotic. Figure 2 shows a comparison of the growth that each bacterial specie had in each medium, namely MRS $+60 \mathrm{~s}$, MRS + $90 \mathrm{~s}, \mathrm{MRS}+30 \mathrm{~s}, \mathrm{MRS}+60 \mathrm{~s}$ fat and MRS + inulin, respectively. For all the strains, except for Yov5, the lowest optical density (OP) was found when inulin was used as their source of carbohydrates while the highest OP corresponded to the medium of the sample 60 sfat. Yov5 did not develop in any medium being its growth equally poor in all conditions with a very low optical density that did not reach 0.2 . Nonetheless, the growth of bacteria in the control medium elaborated with glucose surpassed categorically the growth of the bacteria in the other media. This was expected since this monosaccharide is suited to be readily available for the consumption of the bacteria in a 24-h period of incubation prompting their fast development.

The metabolic preferences of the bacteria used in this experiment could explain their behavior in this study. In general, bacteria of the genus Lactobacillus are Grampositive, non-spore forming, strictly fermentative, microaerophilic and chemoorganotrophic, which means that they require rich media to grow [50] and nowadays constitute the most abundantly used probiotic strains [51]. AL5 and AL3 are two very similar strains that belong to the Lactobacillus gasseri species. These are characterized by having a homofermentative metabolism. They are usually isolated from fecal samples of healthy breast-fed babies and have been extensively studied for their probiotic activities that include pathogen inhibition, epithelial cell attachment, and immunomodulation [52]. Lactobacillus reuteri 17938 (REU) was isolated from a commercial probiotic formulation named Reuterin. It is a heterofermentative bacterium that especially feeds on glucose in order to produce carbon dioxide, ethanol, acetate, and lactic acid. The beneficial effects of oral intake of Lactobacillus reuteri strain DSM 1793 have been well documented in a number of clinical trials [53]. Lactobacillus delbrueckii subsp. bulgaricus is characterized by its versatility of substrates that ferments, being these: lactose, glucose, fructose, and mannose [54]. This microorganism constitutes the starter culture for the production of fermented dairy products.

Within the genus Lactobacillus, and new genera deriving from it, the capacity of individual strains and species for oligosaccharide metabolism substantially differs. The fermentation of carbohydrates is highly variable due to the loss of plasmid encoded traits [55] and gene acquisition by lateral gene transfer [56]. Almost all Lactobacilli metabolize $\alpha$-glucans and many strains possess alternative pathways for metabolism of sucrose and fructooligosaccharides indicating that these are highly preferred substrates. Metabolic pathways for disaccharide metabolism often also enable the metabolism of tri- and tetrasaccharides; nevertheless, metabolic enzymes for oligosaccharide conversion are intracellular which results in the metabolism of oligosaccharide to be limited by transport [57]. Lactobacilli species also distinguish themselves from other common intestinal bacteria as they mainly occupy the first section of large intestine. This situation is a direct reflection of their metabolic preferences towards compounds that are composed of 2 to 10 monosaccharide residues which are abundant in that site, leaving more complex oligosaccharides to pass by to lower parts for the use of Bifidobacterium spp. which have a more 


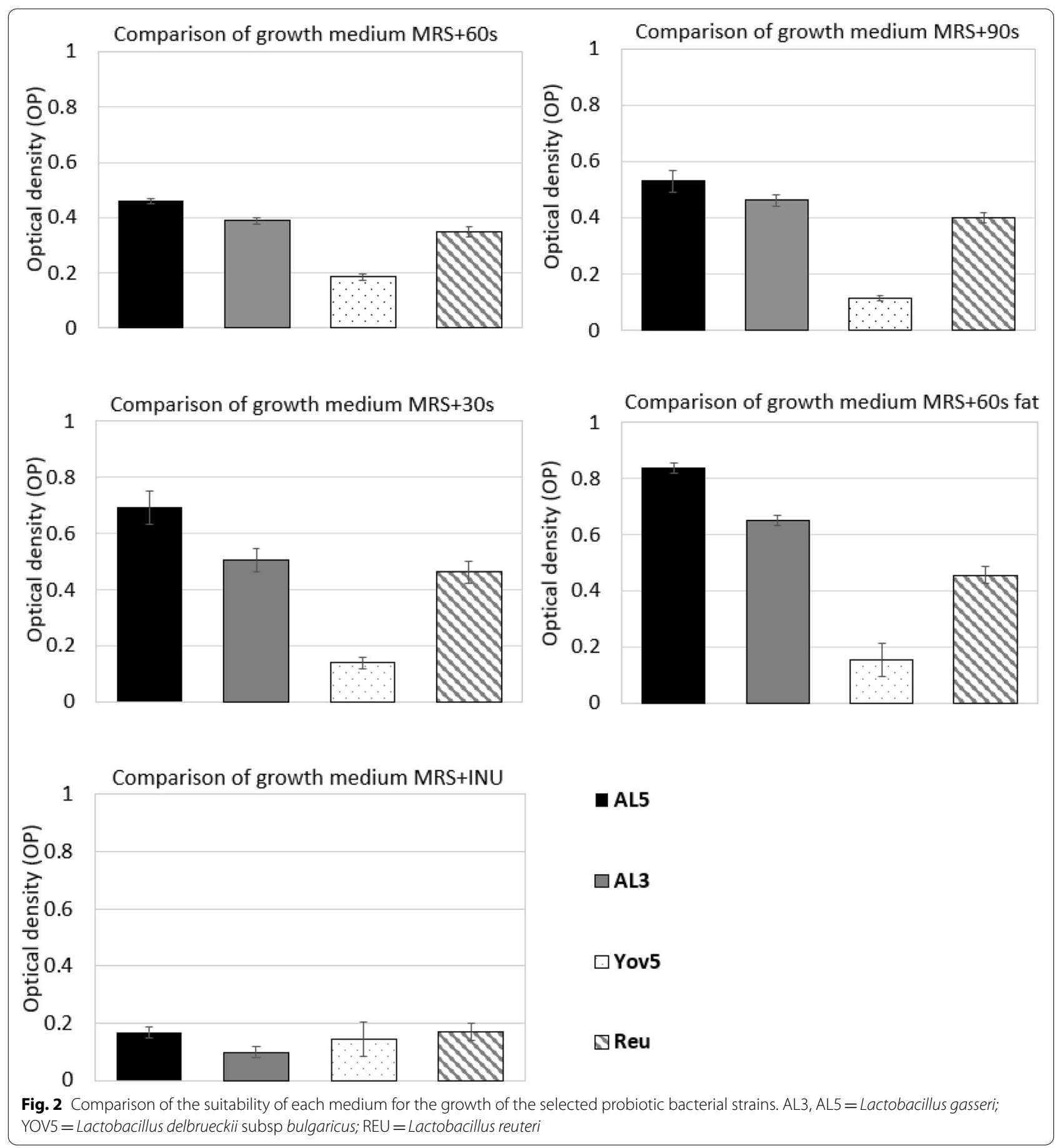

extensive ability for extracellular hydrolysis and transport of complex carbohydrates $[57,58]$.

This preference of substrates of the species is well evidenced in the results of this study in which there was an overall tendency to prefer any of the media prepared with the extracts as opposed to the media prepared with inulin. Inulin is composed of fructose molecules linked by $\beta-(2,1)$ bonds and a terminal glucose residue linked by an $\alpha-(1,2)$ bond with a DP that can range from 10 to 65 fructose units being 12 to 15 the most common values for commercial preparations [59]. This study corroborated that there is a preference of Lactobacillus bacteria for fermenting shorter chains as opposed to longer chains of oligosaccharides. Grootaert et al. [60] contrasted 
arabinoxylan oligosaccharides from cereals with a DP from 3 to 60 against inulin from chicory root and concluded that the length of the chains is an important parameter for prebiotic effects being lower molecular weight molecules more suitable for the growth of some strains. Lopes et al. [59] analyzed fructooligosaccharides and inulin of different degrees of polymerization with six strains of Lactobacilli and concluded that the rate of consumption of these prebiotics increases as the degree of polymerization decreases. Other researchers also tested inulin and other prebiotics on probiotic bacteria concluding that shorter chains were the first to be consumed [42] and that a longer time of incubation is needed before inulin is fermented as opposed to shorter chain oligosaccharides which are readily used in the ascending part of the colon $[60,61]$ and that, in general, some Lactobacilli have a relatively low potential to metabolize higher degree oligosaccharides [62, 63].

Nonetheless, as seen in Fig. 2, the behavior of all the bacteria of this study was not the same, which can be attributed to the high selectivity and particular metabolism of each strain. The fermentation substrates of Lactobacillus gasseri are largely restricted to mono, di, and trisaccharides thanks to the abundance of PTS sugar transporters and galactosidases and the lack of xylanases, amylases, arabinofuranosidases and other enzymes that depolymerize higher order complex polysaccharides [52]. This supports the fact that both strains of this specie developed better than Lactobacillus bulgaricus (Yov5) and Lactobacillus reuteri (Reu) in the three media. In the case of Lactobacillus bulgaricus, it developed equally poor in every medium. Studies have shown that this strain is better at consuming substrates rich in sugars with a degree of polymerization of 5 and 4 units [63]. All the extracts were relatively poor in these oligosaccharides thus their composition was not useful for Yov5. Lactobacillus reuteri did not develop as well as Lactobacillus gasseri either. This strain is characterized by being one of the most representative species that have a narrow spectrum of oligosaccharide fermentation in the intestinal tract implying that it is very selective on the sugars it ferments which might be the reason for its lack of proper development. Furthermore, there was a statistical difference in the growth of AL5 and AL3 in the medium that contained the extract 60sfat whose composition was significantly different from the others. It contained the lowest quantity of oligosaccharides being lower in trisaccharide units but higher in one isomer of tetrasaccharides. Nonetheless, still this sample contained a high amount of trisaccharides (71.60\%), one of the preferred substrates of Lactobacillus gasseri, the better development of the strain can be attributed to the possible presence of other compounds that were not identified in this extract. There is a chance that the effects of the presence of the lipid fraction in the SCG matrix at the time of extraction generated or maintained other compounds that were beneficial for the growth of these strains; however, a deeper analysis of this hypothesis should be further explored in future studies.

\section{Conclusions}

The results obtained in this research represent one of the possible approaches in the valorization of food waste from the coffee industry. It was shown that SCG processed using an acid-catalyzed hydrolysis produced water-soluble extracts containing oligosaccharide chains with a degree of polymerization from 3 to 6 units with prebiotic activity on Lactobacilli strains. The SCG extracts showed to be more effective compared to an established prebiotic such as inulin. Moreover, the effect of inclusion of a prebiotic source into a bacteria growth showed an increased bacterial activity, which depends on the metabolic preferences of the bacteria used in this experiment. Selective processing parameters of acid hydrolysis could determine a different composition of extracted galactooligosaccharides and mannooligosaccharides, tailoring the prebiotic solution to specific bacterial strain. The combination of probiotics with synergistically acting prebiotic substrates would improve bacterial vitality providing a good basis for the development of novel synbiotics (combination of probiotics and prebiotics).

\section{Acknowledgements}

University of Salerno is kindly acknowledged for providing publishing support.

\section{Authors' contributions}

FS: methodology, investigation, validation, formal analysis, visualization, writing-original draft, writing-review and editing, project administration. FM: methodology, formal analysis, visualization. ADV: methodology, writingoriginal draft, writing-review and editing. PV: investigation, supervision. GM: conceptualization, methodology, data curation, resources. DM: data curation. ADT: data curation, supervision. EE-J: methodology, investigation, validation, formal analysis, visualization, writing-original draft, writing-review and editing. All authors read and approved the final manuscript.

\section{Funding}

This research did not receive any specific grant from funding agencies in the public, commercial, or not-for profit sectors.

Availability of data and materials

Not applicable.

\section{Declarations}

Ethics approval and consent to participate Not applicable.

Consent for publication Not applicable. 


\section{Competing interests}

The authors declare that they have no known competing financial interests or personal relationships that could have appeared to influence the work reported in this paper.

\section{Author details}

${ }^{1}$ Department of Agricultural Sciences, University of Naples Federico II, 80055 Portici, NA, Italy. ${ }^{2}$ Department of Industrial Engineering of the University of Salerno, 84084 Fisciano, SA, Italy. ${ }^{3}$ Proteomics, Metabolomics \& Mass Spectrometry Laboratory, ISPAAM-CNR, Portici, NA, Italy. ${ }^{4}$ Department of Food \& Nutrition, College of Human Ecology, Hanyang University, Seongdong-gu, Seoul 04763, Republic of Korea.

\section{Received: 29 June 2021 Accepted: 7 October 2021}

Published online: 26 December 2021

\section{References}

1. Caballero $\mathrm{E}$, Soto C. Valorization of agro-industrial waste into bioactive compounds: techno-economic considerations. In: Bastidas-Oyanedel JR, Schmidt JE, editors. Biorefinery. Cham: Springer; 2019. p. 235-52.

2. Cecilia JA, García-Sancho C, Maireles-Torres PJ, Luque R. Industrial food waste valorization: a general overview. In: Bastidas-Oyanedel JR, Schmidt JE, editors. Biorefinery. Cham: Springer; 2019. p. 253-77.

3. Thi NBD, Kumar G, Lin CY. An overview of food waste management in developing countries: current status and future perspective. J Environ Manage. 2015;157:220-9.

4. Dueñas M, García-Estévez I. Agricultural and food waste: analysis, characterization and extraction of bioactive compounds and their possible utilization. Foods. 2020;9:817.

5. Arya SS, Venkatram R, More PR, Vijayan P. The wastes of coffee bean processing for utilization in food: a review. J Food Sci Technol. 2021. https:// doi.org/10.1007/s13197-021-05032-5.

6. Murthy PS, Naidu MM. Sustainable management of coffee industry by-products and value addition - a review. Resour Conserv Recycl. 2012;66:45-58.

7. Esquivel P, Jiménez VM. Functional properties of coffee and coffee byproducts. Food Res Int. 2012;46:488-95.

8. Mussatto SI, Carneiro LM, Silva JPA, Roberto IC, Teixeira JA. A study on chemical constituents and sugars extraction from spent coffee grounds. Carbohyd Polym. 2011;83:368-74.

9. Iriondo-DeHond A, García NA, Fernandez-Gomez B, Guisantes-Batan E, Escobar FV, Blanch GP, Andres MIS, Sanchez-Fortune S, del Castillo MD. Validation of coffee by-products as novel food ingredients. IFSET. 2019;51:194-204.

10. Scully DS, Jaiswal AK, Abu-Ghannam N. An investigation into spent coffee waste as a renewable source of bioactive compounds and industrially important sugars. Bioengineering. 2016. https://doi.org/10.3390/bioen gineering3040033.

11. Jimenez-Zamora A, Pastoriza S, Rufian-Henares JA. Revalorization of coffee by-products. Prebiotic, antimicrobial and antioxidant properties. LWT Food Sci Technol. 2015;61:12-8.

12. Oseguera-Castro KY, Madrid JA, Madrid MJM, García OP, Del Castillo MD, Campos-Vega R. Antioxidant dietary fiber isolated from spent coffee (Coffea arabica L.) grounds improves chronotype and circadian locomotor activity in young adults. Food Funct. 2019;10:4546-56.

13. Vázquez-Sánchez K, Martinez-Saez N, Rebollo-Hernanz M, del Castillo MD, Gaytán-Martínez M, Campos-Vega R. In vitro health promoting properties of antioxidant dietary fiber extracted from spent coffee (Coffee arabica L.) grounds. Food Chem. 2018;261:253-9.

14. Martinez-Saez N, García AT, Pérez ID, Rebollo-Hernanz M, Mesías M, Morales FJ, Martín-Cabrejas MA, del Castillo MD. Use of spent coffee grounds as food ingredient in bakery products. Food Chem. 2017;216:114-22.

15. Castaldo L, Lombardi S, Gaspari A, Rubino M, Izzo L, Narváez A, Ritieni A, Grosso M. In vitro bioaccessibility and antioxidant activity of polyphenolic compounds from spent coffee grounds-enriched cookies. 2021. Foods. https://doi.org/10.3390/foods10081837.

16. Sampaio A, Dragone G, Vilanova M, Oliveira JM, Teixeira JA, Mussatto SI. Production, chemical characterization, and sensory profile of a novel spirit elaborated from spent coffee ground. LWT Food Sci Technol. 2013;54:557-63.

17. Machado E, Mussatto SI, Teixeira J, Vilanova M, Oliveira J. Increasing the sustainability of the coffee agro-industry: spent coffee grounds as a source of new beverages. Beverages. 2018. https://doi.org/10.3390/bever ages4040105.

18. Tian T, Freeman S, Corey M, German JB, Barile D. Chemical characterization of potentially prebiotic oligosaccharides in brewed coffee and spent coffee grounds. J Agric Food Chem. 2017;65:2784-92.

19. Asano I, Hamaguchi K, Fujii S, lino H. In vitro digestibility and fermentation of mannooligosaccharides from coffee mannan. Food Sci Technol Res. 2003:9:62-6.

20. Asano I, Ikeda Y, Fujii S, lino H. Effects of mannooligosaccharides from coffee on microbiota and short chain fatty acids in rat cecum. Food Sci Techno Res. 2004;10:273-7.

21. Asano I, Umemura M, Fujii S, Hoshino $\mathrm{H}$, lino $\mathrm{H}$. Effects of mannooligosaccharides from coffee mannan on fecal microflora and defecation in healthy volunteers. Food Sci Technol Res. 2004;10:93-7.

22. Panzella L, Pérez-Burillo S, Pastoriza S, Martín MÁ, Cerruti P, Goya L, Ramos S, Rufián-Henares JÁ, Napolitano A, d'Ischia M. High antioxidant action and prebiotic activity of hydrolyzed spent coffee grounds (HSCG) in a simulated digestion-fermentation model: toward the development of a novel food supplement. J Agric Food Chem. 2017;65:6452-9.

23. Wongsiridetchai C, Jonjaroen V, Sawangwan T, Charoenrat T, Chantorn S. Evaluation of prebiotic mannooligosaccharides obtained from spent coffee grounds for nutraceutical application. LWT Food Sci Technol. 2021. https://doi.org/10.1016/j.Iwt.2021.111717.

24. Vandeputte D, Falony G, Vieira-Silva S, Wang J, Sailer M, Theis S, Verbeke $K$, Raes J. Prebiotic inulin-type fructans induce specific changes in the human gut microbiota. Gut. 2017. https://doi.org/10.1136/ gutjnl-2016-313271.

25. Yen W, Wang B, Chang L, Duh P. Antioxidant properties of roasted coffee residues. J Agric Food Chem. 2005;53:2658-63.

26. Fulger CV, Stahl HD, Turek EJ, Bayha R. Production of a Mannan Oligomer Hydrolysate. United States Patent and Trademark Office. 1985. Patent No. 4,508,745.

27. Bajad SU, Lu W, Kimball EH, Yuan J, Peterson C, Rabinowitz JD. Separation and quantitation of water soluble cellular metabolites by hydrophilic interaction chromatography-tandem mass spectrometry. J Chromatogr. 2006;1125:76-88.

28. De Prisco A, van Valenberg HGF, Fogliano V, Mauriello G. Microencapsulated starter culture during yoghurt manufacturing, effect on technological features. Food Bioprocess Technol. 2017;10:1767-77.

29. Maresca D, Zotta T, Mauriello G. Adaptation to aerobic environment of Lactobacillus johnsonii/gasseri strains. Front Microbiol. 2018;9:1-11.

30. Maresca D, De Filippis F, de Tytgat HLP, Vos WM, Mauriello G. Draft Genome sequences of the aerobic strains Lactobacillus gasseri AL3 and AL5. Genome Announc. 2017;5:1-2.

31. Sachslehner A, Foidl G, Foidl N, Gübitz NG, Haltrich D. Hydrolysis of isolated coffee mannan and coffee extract by mannanases of Sclerotium rolfsii. J Biotechnol. 2000;80:127-34.

32. van Dam JEG, Harmsen P. Coffee residues utilization. Wageningen UR, Food \& Biobased Research; 2010. https://edepot.wur.nl/382065.

33. Ballesteros LF, Teixeira JA, Mussatto SI. Chemical, functional, and structural properties of spent coffee grounds and coffee silverskin. Food Bioproc Tech. 2014;7(12):3493-503.

34. López-Barrera DM, Vázquez-Sánchez K, Loarca-Piña MG, Campos-Vega R. Spent coffee grounds, an innovative source of colonic fermentable compounds, inhibit inflammatory mediators in vitro. Food Chem. 2016;212:282-90.

35. Passos CP, Coimbra MA. Microwave superheated water extraction of polysaccharides from spent coffee grounds. Carbohydr Polym. 2013;94(1):626-33.

36. Jooste T, García-Aparicio MP, Brienzo M, van Zyl WH, Görgens JF. Enzymatic hydrolysis of spent coffee ground. Appl Biochem Biotechnol. 2013;169:2248-62.

37. Simões J, Nunes FM, Domingues MR, Coimbra MA. Demonstration of the presence of acetylation and arabinose branching as structural 
features of locust bean gum galactomannan. Carbohydr Polym. 2011:86:1476-83.

38. Nunes FM, Domingues MR, Coimbra MA. Arabinosyl and glucosyl residues as structural features of acetylated galactomannans from green and roasted coffee infusions. Carbohydr Res. 2005;340:1689-98.

39. Nunes FM, Reis A, Silva AMS, Domingues MRM, Coimbra MA. Rhamnoarabinosyl and rhamnoarabinoarabinosyl side chains as structural features of coffee arabinogalactans. Phytochemistry. 2008;69:1573-85.

40. Simões J, Maricato É, Nunes FM, Domingues MR, Coimbra MA. Thermal stability of spent coffee ground polysaccharides: galactomannans and arabinogalactans. Carbohydr Polym. 2014;101:256-64.

41. Borrelli RC, Visconti A, Mennella C, Anese M, Fogliano V. Chemical characterization and antioxidant properties of coffee melanoidins. J Agric Food Chem. 2002;50:6527-33.

42. Takao I, Fujii S, Ishii A, Han L, Kumao T, Ozaki K, Asakawa A. Effects of mannooligosaccharides form coffee mannan on fat storage in mice fed high fat diet. J Health Sci. 2006;52:333-7.

43. Glenn RG, Roberfroid MB. Dietary modulation of the human colonic microbiota: introducing the concept of prebiotics. J Nutr. 1995:125:1401-12.

44. Guarner F, Perdigon G, Corthier G, Salminen S, Koletzko B, Morelli L. Should yoghurt cultures be considered probiotic? Br J Nutr. 2005;93(6):783-6.

45. Mater DD, Bretigny L, Firmesse O, Flores MJ, Mogenet A, Bresson JL, Corthier G. Streptococcus thermophilus and Lactobacillus delbrueckii subsp. bulgaricus survive gastrointestinal transit of healthy volunteers consuming yogurt. FEMS Microbiol Lett. 2005;250(2):185-7.

46. Maya-Barrios A, Lira-Hernandez K, Jiménez-Escobar I, Hernández L, Ortiz-Hernandez A, Jiménez-Gutiérrez C, López-Velázquez G, GutiérrezCastrellón P. Limosilactobacillus reuteri ATCC PTA 5289 and DSM 17938 as adjuvants to improve evolution of pharyngitis/tonsillitis in children: randomised controlled trial. Benef Microbes. 2021;12(2):137-45.

47. Satia I, Cusack R, Stevens C, Schlatman A, Wattie J, Mian F, Killian KJ, O'Byrne PM, Bienenstock J, Forsythe P. Limosilactobacillus reuteri DSM17938 for preventing cough in adults with mild allergic asthma: a double-blind randomized placebo-controlled cross-over study. Clin Exp Allergy. 2021;51(9):1133-43.

48. Saviano A, Brigida M, Migneco A, Gunawardena G, Zanza C, Candelli M, Franceschi F, Ojetti V. Lactobacillus Reuteri DSM 17938 (Limosilactobacillus reuteri) in diarrhea and constipation: two sides of the same coin? Medicina. 2021;57(7):643.

49. Selle K, Klaenhammer TR. Genomic and phenotypic evidence for probiotic influences of Lactobacillus gasseri on human health. FEMS Microbiol Rev. 2013:37(6):915-35.

50. Von Wright A, Axelsson L. Lactic acid bacteria: an introduction. In: Lahtinen S, Ouwehand A, Salminen S, Von Wright A, editors. Lactic acid bacteria: microbiological and functional aspects. Boca Raton: Taylor and Francis Group; 2012. p. 1-16.

51. Shen X, Yi D, Ni X, Zeng D, Jing B, Lei M, Bian Z, Zeng Y, Li T, Xin J. Effects of Lactobacillus plantarum on production performance, immune characteristics, antioxidant status, and intestinal microflora of bursin-immunized broilers. Can J Microbiol. 2014;60:193-202.
52. Pridmore RD, Berger B, Desiere F, Vilanova D, Barretto C, Pittet AC, Zwahlen MC, Rouvet M, Altermann E, Barrangou R, Mollet B, Mercenier A, Klaenhammer T, Arigoni F, Schell MA. The genome sequence of the probiotic intestinal bacterium Lactobacillus johnsonii NCC 533. Proc Natl Acad Sci USA. 2004;101:2512-7.

53. Saulnier DM, Santos F, Roos S, Mistretta TA, Spinler JK, Molenaar D, Teusink $B$, Versalovic J. Exploring metabolic pathway reconstruction and genomewide expression profiling in Lactobacillus reuteri to define functional probiotic features. PLOS ONE. 2011;6:1-14.

54. Chervaux C, Ehrlich SD, Maguin E. Physiological study of Lactobacillus delbrueckii subsp. bulgaricus strains in a novel chemically defined medium. Appl Environ Microbiol. 2000;66(12):5306-11.

55. de Vos P, Faas MM, Spasojevic M, Sikkema J. Encapsulation for preservation of functionality and targeted delivery of bioactive food components. Int Dairy J. 2010;20:292-302.

56. Barrangou R, Altermann E, Hutkins R, Cano R, Klaenhammer TR. Functional and comparative genomic analyses of an operon involved in fructooligosaccharide utilization by Lactobacillus acidophilus. PNAS. 2003;100:8957-62.

57. Gänzle MG, Follador R. Metabolism of oligosaccharides and starch in lactobacilli: a review. Front Microbiol. 2012;3:1-15.

58. Sela DA, Chapman J, Adeuya A, Kim JH, Chen F, Whitehead TR, Lapidus A, Rokhsar DS, Lebrilla CB, German JB, Price NP, Richardson PM, Mills DA. The genome sequence of Bifidobacterium longum subsp. infantis reveals adaptations for milk utilization within the infant microbiome. Proc Natl Acad Sci USA. 2008;105:18964-9.

59. Lopes SM, Krausová G, Rada V, Gonçalves JE, Gonçalves RA, de Oliveira AJ. Isolation and characterization of inulin with a high degree of polymerization from roots of Stevia rebaudiana (Bert.). Bertoni Carbohydr Res. 2015;411:15-21.

60. Grootaert C, Van den Abbeele P, Marzorati M, Broekaert WF, Courtin CM, Delcour JA, Verstraete W, Van de Wiele T. Comparison of prebiotic effects of arabinoxylan oligosaccharides and inulin in a simulator of the human intestinal microbial ecosystem. FEMS Microbiol Ecol. 2009;69:231-42.

61. Van de Wiele T, Boon N, Possemiers S, Jacobs H, Verstraete W. Inulin-type fructans of longer degree of polymerization exert more pronounced in vitro prebiotic effects. J Appl Microbiol. 2007;102:452-60.

62. Ito H, Takemura N, Sonoyama K, Kawagishi H, Topping DL, Conlon MA, Morita T. Degree of polymerization of inulin-type fructans differentially affects number of lactic acid bacteria, intestinal immune functions, and immunoglobulin A secretion in the rat cecum. J Agric Food Chem. 2011;59:5771-8.

63. Mandadzhieva T, Ignatova-Ivanova T, Kambarev S, Iliev I, Ivanova I. Utilization of different prebiotics by Lactobacillus Spp. and Lactococcus Spp. Biotechnol Biotechnol Equip. 2011;25:117-20.

\section{Publisher's Note}

Springer Nature remains neutral with regard to jurisdictional claims in published maps and institutional affiliations.

\section{Submit your manuscript to a SpringerOpen ${ }^{\circ}$ journal and benefit from:}

- Convenient online submission

- Rigorous peer review

- Open access: articles freely available online

- High visibility within the field

- Retaining the copyright to your article

Submit your next manuscript at $\boldsymbol{\nabla}$ springeropen.com 